\title{
OPEN Glycosaminoglycan biosynthesis pathway in host genome is associated with Helicobacter pylori infection
}

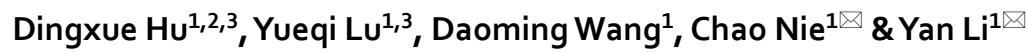

Helicobacter pylori is a causative pathogen of many gastric and extra-gastric diseases. It has infected about half of the global population. There were no genome-wide association studies (GWAS) for $H$. pylori infection conducted in Chinese population, who carried different and relatively homogenous strain of $H$. pylori. In this work, we performed SNP (single nucleotide polymorphism)-based, genebased and pathway-based genome-wide association analyses to investigate the genetic basis of host susceptibility to $H$. pylori infection in 480 Chinese individuals. We also profiled the composition and function of the gut microbiota between $H$. pylori infection cases and controls. We found several genes and pathways associated with $H$. pylori infection $(P<0.05)$, replicated one previously reported SNP rs10004195 in $T L R 1$ gene region $(P=0.02)$. We also found that glycosaminoglycan biosynthesis related pathway was associated with both onset and progression of $H$. pylori infection. In the gut microbiome association study, we identified 2 species, 3 genera and several pathways had differential abundance between $H$. pylori infected cases and controls. This paper is the first GWAS for $H$. pylori infection in Chinese population, and we combined the genetic and microbial data to comprehensively discuss the basis of host susceptibility to $H$. pylori infection.

More than half of the world population were infected by Helicobacter pylori ${ }^{1}$. It is the major cause of gastritis $(80 \%)$ and gastroduodenal ulcer disease (15-20\%) and the only bacterial pathogen believed to cause gastric cancer $^{2,3}$. The prevalence of $H$. pylori infection has large amounts of variations among countries. One of the causes may be that $H$. pylori populations are very diverse, due to point mutations and larger substitutions, insertions or deletions that may involve one or more genes or multigene segments (including restriction/modification genes and at least one pathogenicity island). Divergence among $H$. pylori strains would be further increased by differences among people in traits important to individual strains ${ }^{4}$. Numerous epidemiological studies have investigated the associations between polymorphic genetic elements of $H$. pylori and gastric disease development ${ }^{5,6}$. One study found that gastric cancer was associated with the presence of $b a b A$ and genes in the cag pathogenicity island of $H$. pylori through comparing the strains isolated from gastric cancer patients and gastritis individuals ${ }^{5}$.

In addition to the variation of $H$. pylori genome, the host genetic variation also contributed to the $H$. pylori susceptibility ${ }^{7,8}$, the reported heritability was $57 \%^{8}$. So far, only one genome-wide association study (GWAS) of H. pylori infection was performed in European population ${ }^{9}$. Genetic variations in the toll-like receptor (TLR) locus were found to be associated with $H$. pylori seroprevalence. TLRs are important pattern recognition receptors on cellular surfaces in the innate immune system ${ }^{10}$, known to be essential in providing protective immunity against infection. Many studies reported that TLRs were associated with $H$. pylori infection ${ }^{11,12}$. On the $H$. pylori side, the immune response is triggered by bacterial membrane components, including lipopolysaccharides and lipid A, as well as cytotoxins and $H$. pylori urease activity ${ }^{13}$. Several genetic studies have demonstrated that the variants in $I L 1 B$ were associated with susceptibility to gastric cancer among $H$. pylori infected individuals, this suggested that immune-related genes may be involved in $H$. pylori infection ${ }^{14,15}$. However, there is no GWAS for H. pylori infection on Chinese population.

On the other hand, gut microbiota was reported to play a role in regulating host metabolic function, protecting against pathogen infections and promoting immune system maturation. Previous studies reported some alterations of gut microbiota in $H$. pylori infected patients ${ }^{16,17}$. But there is a lack of research putting genetic

${ }^{1}$ BGI-Shenzhen, Shenzhen 518083, China. ${ }^{2}$ College of Life Sciences, University of Chinese Academy of Sciences, Beijing 100049, China. ${ }^{3}$ These authors contributed equally: Dingxue Hu and Yueqi Lu. ${ }^{\square}$ email: niechao@ genomics.cn; liyan@genomics.cn 


\begin{tabular}{|l|l|l|l|l|l|l|l|}
\hline & \multirow{2}{*}{ Sumber (N) } & Sex & Age & \multicolumn{2}{l|}{ BMI } \\
\cline { 3 - 8 } & female count (percent) & Male count (percent) & Mean (SD) & Range & Mean (SD) & Range \\
\hline Case & 224 & $127(56.70 \%)$ & $97(43.30 \%)$ & $30.78(5.46)$ & $22-58$ & $22.01(3.00)$ & $15.20-30.60$ \\
\hline Control & 256 & $136(53.13 \%)$ & $120(46.88 \%)$ & $31.18(5.40)$ & $22-69$ & $22.25(3.22)$ & $15.70-37.80$ \\
\hline Total & 480 & $263(54.79 \%)$ & $217(45.21 \%)$ & $30.99(5.46)$ & $22-69$ & $22.14(3.12)$ & $15.20-37.80$ \\
\hline
\end{tabular}

Table 1. Characteristic statistics of the study population. After Wilcoxon test, there was no significance in age difference between case and control. In the logistic regression model, H. pylori infection status $\sim$ sex, we found there was no significant correlation between sex and H. pylori infection. BMI had no significant difference in the cases and controls.

susceptibilities and gut microbiome study together to integrate more information for understanding the mechanism of $H$. pylori infection.

Therefore, we carried out a GWAS on $H$. pylori infection and also compared the profiles of gut microbiota between cases and controls with the aim of identifying genetic variants influencing $H$. pylori infection and gaining more insights into the mechanism of $H$. pylori in Chinese population.

\section{Results}

Study sample. $224 \mathrm{H}$. pylori infected positive individuals and 256 negative individuals were selected from an existing data set with the results of carbon-13 urea breath test (C13 test) for $H$. pylori infection. The written form of consent was signed for each individual. The $\mathrm{C} 13$ test results include diagnosis to $H$. pylori infection and the delta over baseline (DOB) values for cases. The distribution of DOB values for cases was shown in Supplementary Fig. S1. The detailed characteristic statistics of the samples were described in Table 1 . The case and control groups were designed to match age, sex and body mass index (BMI). There were no significant differences between two groups in age, sex and BMI.

Association analysis on $H$. pylori infection status and DOB measurements. In order to identify genetic loci involved in $H$. pylori infection, we constructed two models in GWAS after standard quality control (QC) processes. Logistic regression was performed in 224 cases and 256 controls and linear regression was performed in 224 cases. The scree plot of principal component analysis (PCA) was shown in Supplementary Fig. S2a. The top 2 components could explain most of the variations of the population and no clear population stratification was observed (Supplementary Fig. S2b). The variant-based associations showed no genome-wide significant single nucleotide polymorphisms (SNPs) $(P<5 \mathrm{e}-8)$. There were still SNPs reached suggestive level $(P<1 \mathrm{e}-5)$ (Fig. 1), 10 SNPs for logistic regression and 59 SNPs for linear regression (Supplementary Table S1 and Supplementary Table S2). The Q-Q plots of both associations were shown in Supplementary Fig. S3. The inflation factor was 1.00445 for case/control analysis and 1.01076 for DOB values association, indicating there were no population stratification for the analyses.

Then we further investigated the regions harbor suggestive significant SNPs. In case/control association, two signals were located in genes LINC02011 (Fig. 2a) and CACNA1B (Fig. 2b) regions, another signal was located in intergenic region between NEDD1 and RMST (Supplementary Table S1 and Supplementary Fig. S4). Copy number variation in LINC02011 region has been reported to be associated with autism spectrum disorder ${ }^{18,19}$. This region also harbored another non-coding gene FGD5-AS1, it was reported to regulate gastric cancer cell proliferation and chemoresistance ${ }^{20}$. Protein encoded by gene $C A C N A 1 B$ was involved in calcium channel activity. This gene has been found recurrently mutated in 2 or more tumor tissues of 5 gastric cancer patients with $H$. pylori infection ${ }^{21}$. In linear regression based GWAS, 59 SNPs passed the nominal significance level $(P<1 \mathrm{e}-5)$. The signals were located in genes SLC25A3P1, DMRTB1, ST6GALNAC3, KIDINS220, DPP10, CELF2, CCDC77, FTO, ATP2C2-AS1 and LARGE1 regions and some intergenic regions. Their summary statistics were shown in Supplementary Table S2 and Supplementary Fig. S5. Among these regions, FTO (Fig. 2c) was strongly associated with obesity and $\mathrm{BMI}^{22}$. Obesity has been reported to be associated with $H$. pylori infection ${ }^{23}$. It is also a risk factor for gastric cancer ${ }^{24}$. One study found that gastric cancer tissues had high FTO expression as compare with the adjacent non-cancerous tissues ${ }^{25}$. LARGE1 gene (Fig. 2d) was involved in immune response for disease pathogens ${ }^{26}$.

Set-based association for further identification of susceptibility genes and pathways for $\boldsymbol{H}$. pylori infection. To improve GWAS power, set-based analyses were performed, which combined the GWAS summary statistics of all variants within a putative biologically functional unit (coding region and possible regulatory region, genes within one pathway) to obtain a single $P$ value that represented the significance level of the associations between $H$. pylori infection and the functional unit. Our SNPs were mapped onto 25,928 genes for gene-based association. The top associated genes for case/control comparison were LOC105376331 and LINC02011 (gene-level $P<1 \mathrm{e}-04$ ). And the most significant genes for linear regression were LARGE1 and LINC00561 (gene-level $P<1 \mathrm{e}-04$ ). They failed to pass genome-wide gene-based significance threshold $(0.05 / 25928)$. Then we further mapped these genes to canonical pathways from molecular signature database ${ }^{27,28}$.

164 pathways were included in the analyses. Top pathways associated with $H$. pylori infection $(P<0.05)$ were listed in Tables 2 and 3. In the logistic model-based pathway association, the glycosphingolipid biosynthesis related pathway (glycosphingolipid biosynthesis-lacto and neolacto series) was the most significant pathway. 

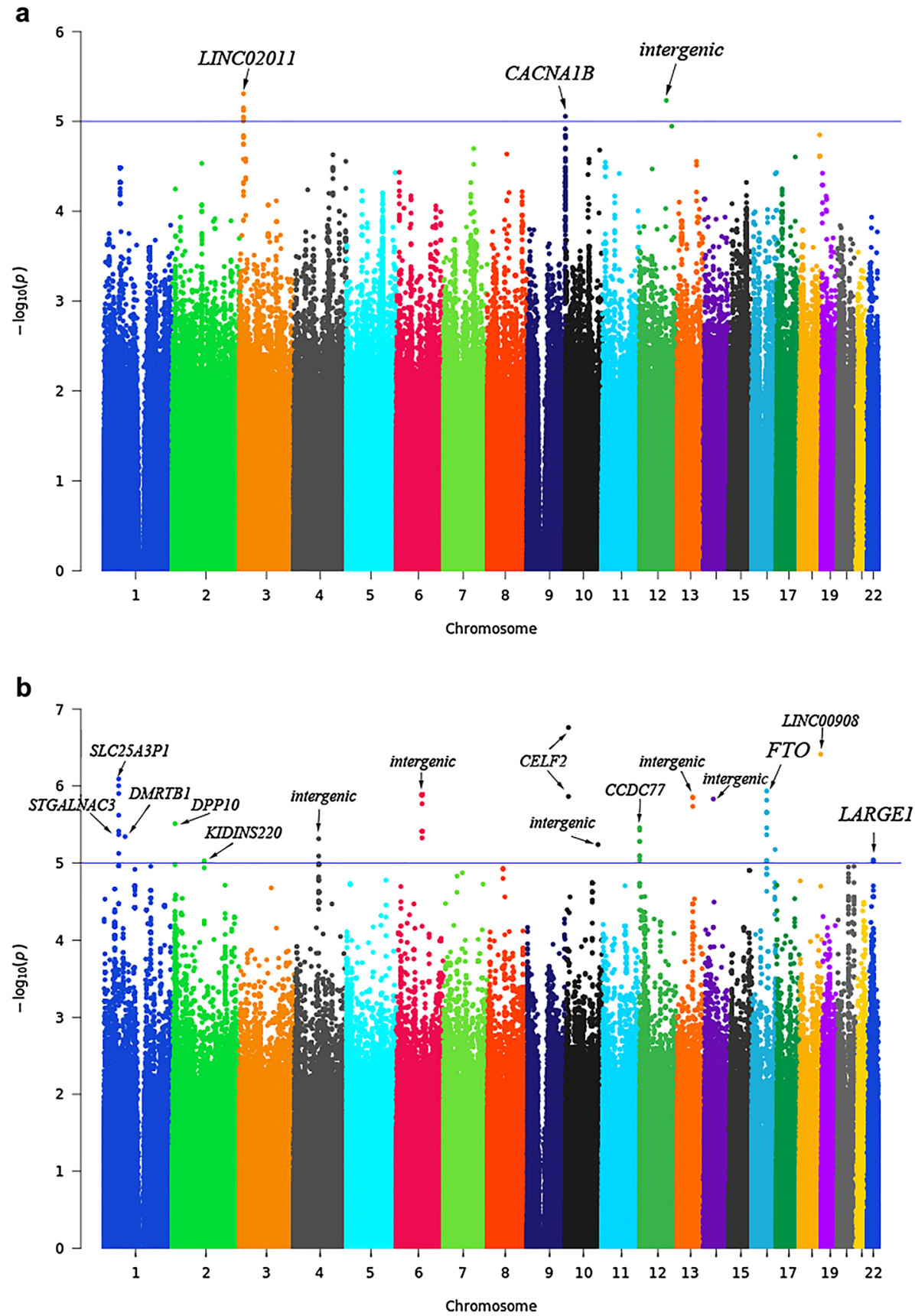

Figure 1. Manhattan plots of genome-wide association analyses. (a) Manhattan plots presenting the $-\log _{10} P$ values from the GWAS adjusted for the top two principal components of the $H$. pylori infection cases versus all controls and (b) the DOB values in $H$. pylori infection cases. The blue line indicates the threshold for suggestive significant association $(P<1 \mathrm{e}-5)$. The genes that suggestive significant variants encoded are marked in the plot.

One study reported that after $H$. pylori eradicated successfully, glycosphingolipid biosynthesis_lacto and neolacto series pathway was significantly altered ${ }^{29}$. In the linear model-based pathway association, the most significant pathway was antigen processing and presentation pathway. Glycosaminoglycan biosynthesis of heparan sulfate (HS) pathway was significant in the both two models, it was a pathway correlated with bacterial adherence ${ }^{30}$. Several immune-related pathways were ranked top in the pathway-based association, indicating the variation of immune systems leading to different infection status of $H$. pylori infection. Folate biosynthesis pathway was also significantly correlated with different infection status of $H$. pylori infection. Study reported that folate level was significantly decreased (18.6\%) in $H$. pylori infected individuals than those who were not infected ${ }^{31}$.

Replication of previous reported $\boldsymbol{H}$. pylori infection related genes. We have checked the two signals reported in previous GWAS in European population'. The rs10004195 in gene TLR1 was replicated in 
a

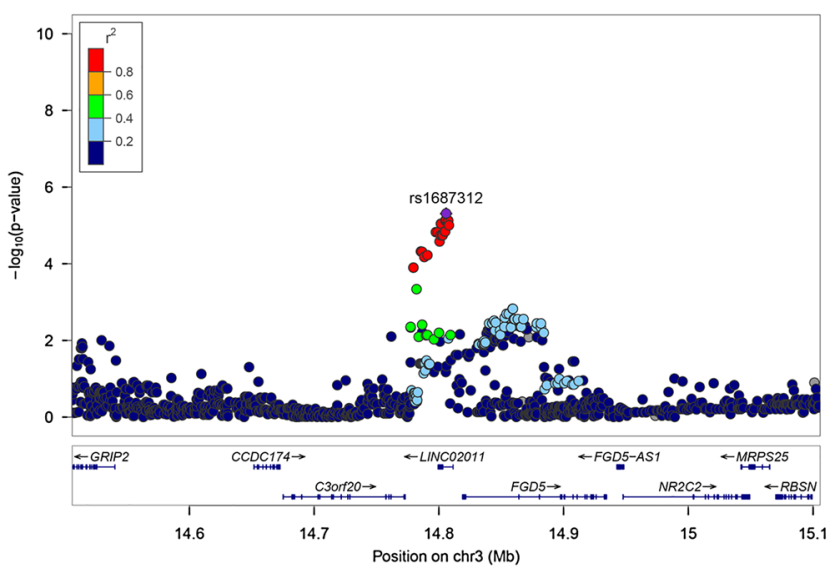

C

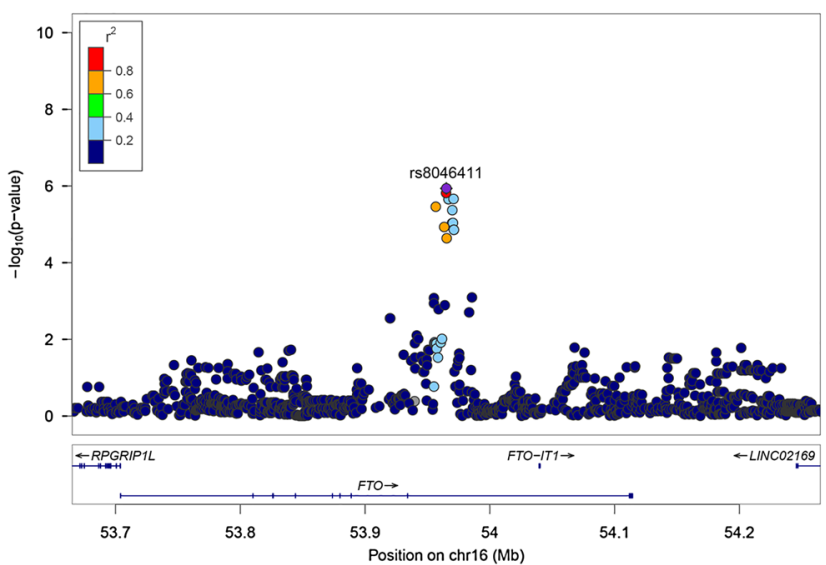

b

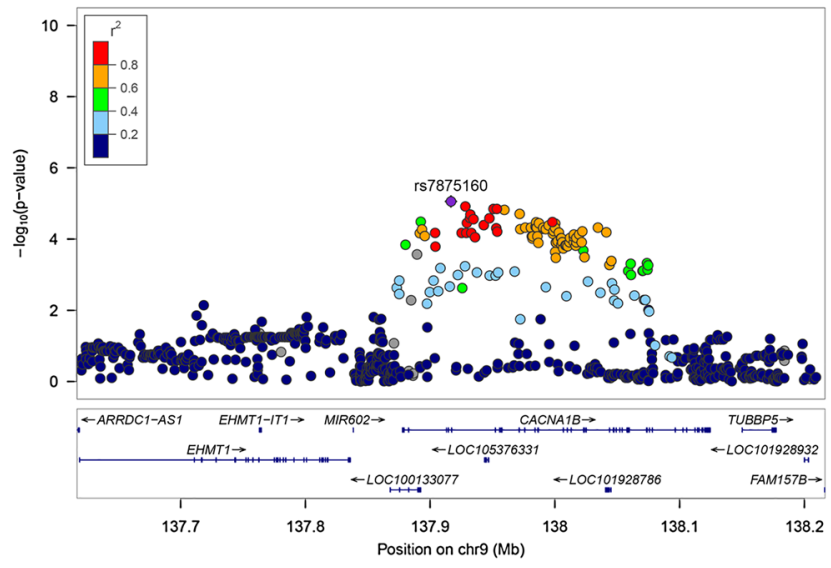

d

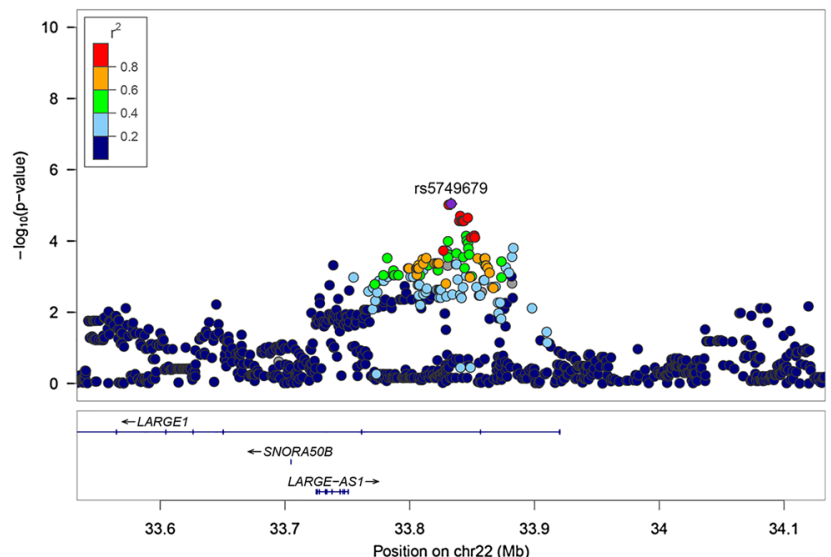

Figure 2. Regional association plots for the regions containing associated genes. Regional association plots for the LINC02011 (a), CACNA1B (b), FTO (c) and LARGE1 (d). LINC02011 (a) and CACNA1B (b) were identified based on the GWAS results from the H. pylori infection cases versus controls, FTO (c) and LARGE1 (d) were identified based on the DOB values association study of $H$. pylori infection cases. The color of the variants is based on the linkage disequilibrium with rs1687312 (LINC02011) (a), rs7875160 (CACNA1B) (b), rs8046411 (FTO) (c) and rs5749679 (LARGE1) (d). For all plots, each point represents a SNP, where the $\mathrm{x}$ axis represents the position of the SNPs and the $y$ axis represents the $-\log 10 P$ values of the GWAS results. Each point is colorcoded with the $\mathrm{r}^{2}$ value as calculated with the source of LD information retrieved from hg38/1000 Genomes Nov 2014 EAS (Asian). Plots showed the most significant SNPs flank $300 \mathrm{~kb}$.

\begin{tabular}{|l|l|l|l|l|l|}
\hline Pathway set & No.SNPs & Chisq(Obs) & P value & TopSNP.Pvalue & TopSNP \\
\hline $\begin{array}{l}\text { KEGG_GLYCOSPHINGOLIPID_BIOSYNTHESIS_LACTO_ } \\
\text { AND_NEOLACTO_SERIES }\end{array}$ & 18 & 58.7025 & $2.03 \mathrm{E}-02$ & $9.41 \mathrm{E}-03$ & rs872451 \\
\hline $\begin{array}{l}\text { KEGG_GLYCOSAMINOGLYCAN_BIOSYNTHESIS_HEP- } \\
\text { ARAN_SULFATE }\end{array}$ & 80 & 149.922 & $2.50 \mathrm{E}-02$ & $3.82 \mathrm{E}-03$ & rs7760512 \\
\hline KEGG_CYTOSOLIC_DNA_SENSING_PATHWAY & 14 & 37.2182 & $3.88 \mathrm{E}-02$ & $7.01 \mathrm{E}-03$ & rs118156647 \\
\hline KEGG_AXON_GUIDANCE & 120 & 207.521 & $4.33 \mathrm{E}-02$ & $1.90 \mathrm{E}-03$ & rs12572571 \\
\hline $\begin{array}{l}\text { KEGG_NATURAL_KILLER_CELL_MEDIATED_CYTOTOX- } \\
\text { ICITY }\end{array}$ & 25 & 58.616 & $4.68 \mathrm{E}-02$ & $6.96 \mathrm{E}-03$ & rs13420683 \\
\hline KEGG_PRIMARY_IMMUNODEFICIENCY & 25 & 58.616 & $4.68 \mathrm{E}-02$ & $6.96 \mathrm{E}-03$ & rs13420683 \\
\hline KEGG_FC_GAMMA_R_MEDIATED_PHAGOCYTOSIS & 4 & 10.6895 & $4.90 \mathrm{E}-02$ & $3.88 \mathrm{E}-02$ & rs57919378 \\
\hline KEGG_RNA_DEGRADATION & 16 & 37.3961 & $4.96 \mathrm{E}-02$ & $6.68 \mathrm{E}-03$ & rs34113220 \\
\hline
\end{tabular}

Table 2. Candidate pathways associated with $H$. pylori infection in logistic regression model. No.SNPs: number of SNPs in the pathway; Chisq(Obs): sum of chi-squared test-statistics of all SNPs in the set; $P$ value: pathway set-based test $P$ value; TopSNP.Pvalue: smallest single-SNP GWAS $P$ value in the pathway set; TopSNP: the top associated GWAS SNP. The rsID is based on dbSNP build 138 . 


\begin{tabular}{|l|l|l|l|l|l|}
\hline Pathway set & No.SNPs & Chisq(Obs) & P value & TopSNP.Pvalue & TopSNP \\
\hline KEGG_ANTIGEN_PROCESSING_AND_PRESENTATION & 46 & 126.12 & $9.74 \mathrm{E}-03$ & $1.03 \mathrm{E}-03$ & rs 12204338 \\
\hline KEGG_AUTOIMMUNE_THYROID_DISEASE & 46 & 126.12 & $9.74 \mathrm{E}-03$ & $1.03 \mathrm{E}-03$ & rs 12204338 \\
\hline KEGG_HEDGEHOG_SIGNALING_PATHWAY & 6 & 23.2668 & $1.60 \mathrm{E}-02$ & $5.35 \mathrm{E}-03$ & rs9576171 \\
\hline KEGG_TERPENOID_BACKBONE_BIOSYNTHESIS & 9 & 32.6463 & $1.91 \mathrm{E}-02$ & $8.99 \mathrm{E}-03$ & rs61836189 \\
\hline $\begin{array}{l}\text { KEGG_NEUROACTIVE_LIGAND_RECEPTOR_INTERAC- } \\
\text { TION }\end{array}$ & 29 & 70.052 & $2.28 \mathrm{E}-02$ & $1.56 \mathrm{E}-03$ & $\mathrm{rs} 2146490$ \\
\hline KEGG_ADIPOCYTOKINE_SIGNALING_PATHWAY & 19 & 48.4956 & $2.97 \mathrm{E}-02$ & $2.09 \mathrm{E}-02$ & rs 12602449 \\
\hline KEGG_JAK_STAT_SIGNALING_PATHWAY & 19 & 48.4956 & $2.97 \mathrm{E}-02$ & $2.09 \mathrm{E}-02$ & rs 12602449 \\
\hline KEGG_FOLATE_BIOSYNTHESIS & 21 & 46.2834 & $4.66 \mathrm{E}-02$ & $1.16 \mathrm{E}-02$ & rs 13131273 \\
\hline $\begin{array}{l}\text { KEGG_GLYCOSAMINOGLYCAN_BIOSYNTHESIS_HEP- } \\
\text { ARAN_SULFATE }\end{array}$ & 80 & 135.496 & $4.77 \mathrm{E}-02$ & $5.04 \mathrm{E}-03$ & rs12195272 \\
\hline
\end{tabular}

Table 3. Candidate pathways associated with $H$. pylori infection in linear regression model. No.SNPs: number of SNPs in the pathway; Chisq(Obs): sum of chi-squared test-statistics of all SNPs in the set; $P$ value: pathway set-based test $P$ value; TopSNP.Pvalue: smallest single-SNP GWAS $P$ value in the pathway set; TopSNP: the top associated GWAS SNP. The rsID is based on dbSNP build 138.

\begin{tabular}{|l|l|l|l|l|l|}
\hline \multirow{2}{*}{ Taxo } & \multirow{2}{*}{ Mean difference } & P value & FDR-adjusted P & HPI negative & HPI positive \\
\hline Holdemanella biformis & 0.0008 & 0.0004 & 0.0579 & 0.2156 & 0.3838 \\
\hline Catenibacterium mitsuokai & 0.0002 & 0.0010 & 0.0785 & 0.0596 & 0.1622 \\
\hline 'Erysipelotrichaceae noname' & 0.0008 & 0.0003 & 0.0212 & 0.4266 & 0.5946 \\
\hline Catenibacterium & 0.0002 & 0.0010 & 0.0349 & 0.0596 & 0.1622 \\
\hline Prevotella & 0.0648 & 0.0037 & 0.0861 & 0.8716 & 0.8973 \\
\hline
\end{tabular}

Table 4. Bacteria associated with $H$. pylori infection in Wilcoxon test. Taxo: identified bacteria names at last taxonomic level by MetaPhlAn2; Mean difference: difference of bacteria abundance between $H$. pylori infection positive group and $H$. pylori infection negative group; $P$ value: $P$ value of Wilcoxon test; FDR: taxonomic level-based FDR adjusted $P$ value; Presence ratio: ratio of non-zero abundance individuals to all people, HPI negative: $H$. pylori infection negative; HPI positive: $H$. pylori infection positive.

the linear association study $(P=0.02)$, of which the minor allele $\mathrm{T}$ has reduced effects on $H$. pylori infection. The SNP rs368433 was filtered out by our QC analysis because it is a low-frequency SNP in Asian (dbSNP: minor allele frequency $(\mathrm{MAF})=0.009)$. The SNP rs10004195 was located in a region which included multiple TLRs (TLR10, TLR1 and TLR6). TLRs play crucial roles in activating innate immunity. The SNP rs368433 was located in FCGR2A gene region. Further gene-based association combining the information from neighboring SNPs within a region showed that TLRs region was replicated $(P<0.05)$ based on the linear regression results. FCGR2A was not replicated $(P>0.05)$.

Differences of gut microbiomes were identified between $\boldsymbol{H}$. pylori infected groups and controls. Stool samples from 403 individuals were collected and metagenome sequencing was utilized to study the gut microbiome between $185 \mathrm{H}$. pylori infected cases and 218 controls. In total, we identified 961 taxonomic categories from kingdom to species assigned by MetaPhlAn2 ${ }^{32}$. Among them, 782 categories were classified to the kingdom of bacteria and its sub-levels. After removing categories with carriers less than $10 \%, 301$ categories in different taxonomic levels were remained.

Then we compared the differences of bacterial abundance between case and control groups at different taxonomic levels. After false discovery rate (FDR) multiple testing adjustment, the abundance of two species were significantly different, Holdemanella biformis (FDR-adjusted $P=0.0579$ ) and Catenibacterium mitsuokai (FDR-adjusted $P=0.0785$ ) (Table 4). These 2 bacteria categories all demonstrated higher presence ratio in case group rather than control group. H. biformis is one of the species in the family Erysipelotrichaceae which is a key butyrate producing taxa ${ }^{33-35}$. The Erysipelotrichaceae was associated with inflammation-related gastrointestinal diseases ${ }^{36}$. As for C. mitsuokai, its abundance would increase in mice after intervention with a high-fat and highsugar diet $^{37}$, and it was closely related to unhealthy fasting serum lipid profile in obese women ${ }^{38}$. This species is the only known species from the genus Catenibacterium. The abundance of three genera were significantly different, 'Erysipelotrichaceae noname', (FDR-adjusted $P=0.0212$ ), Catenibacterium (FDR-adjusted $P=0.0349$ ), Prevotella (FDR-adjusted $P=0.0861$ ) (Table 4). 'Erysipelotrichaceae noname' is an unidentified genus annotated by MetaPhlAn $2^{32}$ below the family Erysipelotrichaceae mentioned above. Prevotella is one of the bacteria producing acetate while acetic acid participating in cholesterol synthesis ${ }^{39,40}$. The abundance of these categories were higher in the $H$. pylori infected groups. We didn't observe significant differences between $H$. pylori infected cases and controls in terms of alpha and beta diversities of bacteria. The species classified below these three genera were listed in Supplementary Table S3. 
Furthermore, we identified $37 \mathrm{H}$. pylori infection specific metabolic pathways which have different abundance in the case and control groups through logistic regression at nominal significance level $(P<0.05)$ (Supplementary Table S4). These results suggested that pathways involved in amino acids biosynthesis (e.g.: L-isoleucine, L-ornithine were observed in $H$. pylori infection negative individuals, while L-lysine, L-lysine biosynthesis, S-adenosyl-L-methionine cycle I, L-citrulline metabolism and L-histidine degradation pathways had higher abundance in $H$. pylori infection positive groups), nucleotides metabolism (e.g.: pyrimidine related pathways were enriched in $H$. pylori infection negative group, while purine related pathways were enriched in $H$. pylori infection positive groups.), and other energy metabolites (e.g.: pyridoxal 5'-phosphate biosynthesis, glycolysis, homolactic fermentation, mannitol cycle, folate related pathways and peptidoglycan pathways) may be different between $H$. pylori infection case and control groups. Interestingly, we found that folate biosynthesis pathway was associated with $H$. pylori infection in both our genetic and microbiome study. It has been reported that folate level was significantly decreased (18.6\%) in H. pylori infection individuals than those who were not infected in a low-income population in the United States ${ }^{31}$.

\section{Discussion}

In this study, we performed genome-wide association study on $224 \mathrm{H}$. pylori infected individuals and 256 negative individuals and also compared their differences of gut microbiome with the aim of gaining more insights into the bases and consequences of $H$. pylori infection. We identified 3 genetic regions which were associated with $H$. pylori infection status and 14 genetic regions which were related to the bacteria load at suggestive significance level $(P<1 \mathrm{e}-5)$. Further pathway-based analysis showed that several pathways were associated with $H$. pylori infection. Those pathways were related to immune response and glycosylation. One of the two reported loci from previous GWAS analysis was replicated in our data. In gut microbiome analysis, 2 species, 3 genera and several pathways were identified to have different abundance between $H$. pylori infected cases and controls.

While comparing the results with the reported GWAS about $H$. pylori infection in $2013^{9}$, we found that rs10004195 in gene TLR1 was associated with the severity of $H$. pylori infection $(P=0.02023)$, but it was not associated with the infection status. TLRs are known to be essential in providing protective immunity against infection. The minor allele of rs10004195 may be associated with less efficiency of anti-inflammatory TLR1-TLR2 signaling'. The other reported SNP rs368433 was filtered out by QC in our genetic data due to its low frequency in Chinese population (MAF $=0.009$ ).

One possible cause of these different results may be the distinct methods for identifying $H$. pylori infection. In our study, we adopted C13 test to detect H. pylori infection. While in the GWAS for European population ${ }^{9}$, they tested anti-H. pylori serum IgG antibody titers. C13 test has higher sensitivity and specificity than anti$H$. pylori serum IgG antibody titers test ${ }^{41}$. Although, anti- $H$. pylori serum IgG antibody titers could represent previous infection, it can hardly represent the current bacteria load in an individual. Besides, C13 test is a more convenient handling method. Therefore, we have chosen $\mathrm{C} 13$ breath tests in our study to measure both onset and severity of $H$. pylori infection. In addition, the population ancestry differences could be another cause for the variation of GWAS results. SNP rs368433 is common in European population and significantly associated with H. pylori infection ${ }^{9}$. However, it is a low frequency SNP $(\mathrm{MAF}=0.009)$ in Chinese population, and it was filtered out in our QC processes. We also checked the adjacent SNPs whose position are close to rs368433, they were not associated with $H$. pylori infection in our data. The true causal variant could still be hidden in this genomic region, but due to different linkage disequilibrium (LD) structure among European and Chinese populations, the alleles tagging true causal variants may be different. Future fine mapping will be required to extend the region and dig the hidden information.

Another difference for $H$. pylori infection between Chinese and European population is that they carry distinct H. pylori strains ${ }^{4}$. Almost all Asian H. pylori strains contain the complete cag-pathogenicity island (PAI) which was associated with more severe disease in $H$. pylori infected individuals ${ }^{4}$.

When comparing our results to existing functional studies, the functions of our newly identified genes and pathways were highly relevant to $H$. pylori infection. FGD5-AS1 regulates cancer gastric cell proliferation ${ }^{20}$. CAC$N A 1 B$ had accumulated somatic mutations in gastric tumor with $H$. pylori infection ${ }^{21}$. Genetic variants associated with obesity were also ranked top in our results. FTO is one of the leading genes associated with obesity and $\mathrm{BMI}^{42,43}$. Studies also found that gastric cancer tissues had high FTO expression compared with the adjacent noncancerous tissues ${ }^{25}$. Obesity was considered as a risk factor for $H$. pylori infection ${ }^{44}$. Interestingly, differences of obesity related bacteria were also identified in our gut microbiome analysis. C. mitsuokai was enriched in H. pylori infection positive group, which was also enriched in obese women ${ }^{38}$. The associations among obesity, genetics of obesity, obesity related bacteria and $H$. pylori infection were reported pair wisely, but their causal relationships are still largely unknown. It is reported that $H$. pylori persistent infection had a negative effect on the fall of BMI values in Chinese obese population ${ }^{45}$. This comorbidity between $H$. pylori infection and obesity may be caused by the pleiotropic effects of FTO and other genes. We also found that glycometabolism-related genes and pathways were associated with $H$. pylori infection. LARGE1 encoded a glycosylase, which played an important role in host-pathogen interactions ${ }^{46}$. Our pathway-based associations identified that glycosphingolipid biosynthesis and glycosaminoglycan biosynthesis were associated with $H$. pylori infection. Glycosphingolipids (GSLs) are components of cell-surface or basement membrane-associated proteoglycans produced by both epithelial and mesenchymal cells ${ }^{47}$. They are known to play a role as receptors in pathogen invasion ${ }^{48}$. Glycosaminoglycans have been shown to be recognized by $H$. pylori outer-membrane proteins and to be involved in the adhesion of the bacteria in cell-line models ${ }^{30}$. In the functional pathway enrichment of microbiome association study, we found that glycolysis related pathways were correlated with $H$. pylori infection. Many studies found that $H$. pylori can metabolize glucose by both oxidative and fermentative pathways ${ }^{49-53}$. H. pylori infected gastric epithelial cells have exhibited increased glycolysis and increased expression of Lon protease 1 (Lonp1), Lonp1 is a key 
regulator of metabolic reprogramming in $H$. pylori-induced gastric carcinogenesis ${ }^{54,55}$. We also identified two immune-related pathways, including antigen-processing and presentation pathway as well as autoimmune thyroid disease pathway. Study has reported that $H$. pylori affects the antigen presentation activity through microRNA ${ }^{56}$. $H$. pylori infection also has immune evasion strategies. Therefore, it was as expected that the genetic variations of immune-related genes may affect the susceptibilities of $H$. pylori infection.

While extensive number of studies have been focused on the alterations of gastric microbiota ${ }^{29}$ since $H$. pylori mainly colonizes the stomach ${ }^{57}$, little is known about the impact of $H$. pylori on gut microbiota. We have performed association analyses between $H$. pylori infection status and gut microbiome using shotgun metagenome sequencing. Compared to existing 16S rRNA gene sequencing for gut microbiota, shotgun sequencing has more power to identify less abundant taxa than $16 \mathrm{~S}$ sequencing ${ }^{58}$. We identified 2 species, 3 genera and several pathways had differential abundance between $H$. pylori infected cases and controls. Interestingly, we found that folate biosynthesis pathway was associated with $H$. pylori infection in both genetic and microbiome studies. Reduction of vitamin B12 and folate concentrations has been reported in $H$. pylori-infected patients ${ }^{59}$. Folate acid was synthesized by bacteria ${ }^{60}$. Altogether, it is suggested that $H$. pylori infection could alter gut microbiome and thereby influence the nutrition supply of the host. More metabolomic and nutrition studies may be required to study the consequences of $H$. pylori infection.

There are some limitations of current study. The sample size was not large enough to boost the signals to genome-wide significance. We have leveraged on the set-based association by combining the SNPs within a biological meaningful set and generated a summed statistic for the whole set. Our set-based association identified glycosaminoglycan and immune-related pathways, which were functional relevant with $H$. pylori infection. We also studied the gut microbiome differences between $H$. pylori infected cases and controls, the results showed more supporting evidence that the glycosylation related function was involved in $H$. pylori infection. Together, we provided a list of candidate genes, pathways and bacteria associated with $H$. pylori infection for further replication and validation.

\section{Methods}

Sample recruitment and data collection. The samples of current study were selected from a cohort study of Shenzhen local area. Individuals who had C13 test and no symptoms of any gastrointestinal conditions were selected. $\mathrm{C} 13$ test is used for detecting $H$. pylori infection. It involves taking C13 isotope-labeled urea molecules by mouth, and then measuring the changes in carbon dioxide in the exhaled carbon by an isotope mass spectrometer. The main measurement index was the $\delta$ value, which measured the ${ }^{13} \mathrm{C} /{ }^{12} \mathrm{C}$ ratio in the breath samples. DOB value is the difference between post-drug $\delta$ value minus pre-drug $\delta$ value. $H$. pylori infection is considered positive if $\mathrm{DOB} \geq 4$. Otherwise, $H$. pylori infection is negative $(\mathrm{DOB}<4)$. Individuals who were diagnosed as $H$. pylori infection for both year 2017 and 2018 were further considered as the cases in our study. Individuals with $\mathrm{DOB}$ value equal to 0 were defined as control to ensure negative infection. At the end, 480 subjects (female $\mathrm{N}=263$, male $\mathrm{N}=217$ ) were selected for the association analyses.

Whole genome sequencing. The whole blood drawn from the participant vein was stored in the ethylenediaminetetraacetic acid (EDTA) anticoagulant tubes to avoid hemolysis, while the plasma was obtained by centrifugation $(3000 \mathrm{rpm}, 10 \mathrm{~min})$ and was preserved at $-80{ }^{\circ} \mathrm{C}$ until assay. The white cells were isolated for genomic DNA extraction. Whole-genome sequencing (WGS) to 30× were conducted on BGI-seq500 sequencer. WGS data were aligned and variants called by the Picard/BWA ${ }^{61} /$ GATK $^{62}$ pipeline. SNPs with mapping quality greater than 40, sequencing depth greater than 4, variant quality greater than 2.0, Phred score of Fisher's test $P$ value for stand bias smaller than 60.0, haplotype score smaller than 13.0 and distance of alternative allele from the end of reads greater than 8.0 were kept for following analyses.

GWAS quality control. The common practice of GWAS QC is conducted through two dimensions of the data: samples and variants. All QC assessments and successive filtering were done using PLINK1.9 ${ }^{63}$. Samples had more than $1 \%$ missing genotyped SNPs, different genetic sex with the record in the phenotypic database, abnormal autosomal heterozygosity were removed. Sex conflict and abnormal heterozygosity may be caused by DNA sample contamination. One of paired samples who have genetic relationship within three degree of relatedness was filtered out. SNPs had high rate of missing genotypes, and deviated from the Hardy-Weinberg equilibrium (HWE) test $(P \leq 1 \mathrm{e}-05)$ as well as SNPs on X and Y chromosomes and mitochondria were also removed. After variant QC, 5,169,391 common SNPs (MAF>0.05) were including for the association analyses. PCA was performed using SNPs on autosomal chromosome by PLINK1.9 $9^{63}$ to investigate population stratification. No clear sub-cluster was observed. Typical north to south Grandaunt was demonstrated by the first principal component (PC). Linear and logistic regression added top two PCs as covariates.

Single-variant-based association. When phenotypes and genotypes have passed all the QC processes, the statistical analysis of disease associations can be performed. The basic analysis of genome-wide association data is a series of single-locus statistical tests, examining each SNP independently for association to the disease status $^{64}$. The types of statistical tests utilized mostly depend on the types of disease phenotypes. For continuous phenotype, $\mathrm{DOB}$ values of the $\mathrm{C} 13$ test results, additive model into linear regression was performed in 224 cases to identify genes associated with severity of $H$. pylori infection. While for binary trait, the case/control status, additive model into logistic regression was utilized in 224 cases and 256 controls to search for genes related to susceptibility of $H$. pylori. According to the scree plot of PCA (Supplementary Fig. S2), the top 2 PCs could explain most of the variations of the population and no clear population stratification observed (Supplementary Fig. S2). After Wilcoxon test, there was no significant difference for age in case/control and cases. In the logistic 
regression model, $H$. pylori infection status $\sim$ sex, we found there was no significant correlation between sex and H. pylori infection. Therefore, the association analyses were only adjusted the top 2 PCs. The regression model used in DOB values analysis was DOB values in cases $\sim$ Genotype $+\mathrm{PC} 1+\mathrm{PC} 2$. The regression model used in $H$. pylori infection was $H$. pylori status $\sim$ Genotype $+\mathrm{PC} 1+\mathrm{PC} 2$. The adaptive permutation tests were used to generate significance levels empirically. All the analyses were performed in PLINK1.963. The Manhattan plots were generated in R 3.6.1. Regional plots were generated by LocusZoom tool ${ }^{65}$ (http://locuszoom.org/).

Gene/pathway-based association. The standard analysis of genome-wide association study uses single SNP marker as the test unit. However, due to small sample size, the complex LD structures and ethnic differences among different populations, many replication studies have failed. To improve GWAS power, the genebased association has been proposed ${ }^{66}$. The gene-based analysis combined the summary statistics of all variants within a putative gene (coding region and possible regulatory region) to obtain a single $P$ value that represents the significance level of diseases and genes. Similarly, variants within genes on the same pathway were combined for pathway-based analysis. Gene-based and pathway-based analyses were performed using GCTA-fastBAT tool $^{67,68}$. In gene-based analysis, a gene region was defined as $\pm 8 \mathrm{~kb}$ of UTRs of a gene.

Metagenomics sequencing. Fresh stool samples were collected from recruited volunteers for metagenomics sequencing. The fecal DNA extractions were processed following the MetaHIT protocol, then single-end metagenomics sequencing was performed using BGISEQ-500 platform. The low-quality reads were discarded, and the host DNA were removed based on human genome reference (hg38) by SOAP2 ${ }^{69}$ (version 2.22). Taxonomic analysis was performed using MetaPhlAn $2^{32}$ following removal of human reads. The relative abundance of species and genera were used in the current study. Meanwhile, gene family abundance and pathway abundance of each sample were profiled using HUMAnN $2^{70}$ with default parameters. HUMAnN2 $2^{70}$ reported the abundance of gene families from the UniProt Reference Clusters (UniRef90), which were further mapped to microbial pathways from the MetaCyc metabolic pathway database.

Gut microorganisms belonging to Kingdom Bacteria were extracted for correlation analysis between $H$. pylori infection and microbial abundance. Bacteria were excluded if they have less than $10 \%$ carriers in the population. After filtering, the abundance of each bacterium was rescaled into percentage within an individual. Then, Wilcoxon test was used to compare the abundance of bacteria between $H$. pylori cases and controls. The $P$ values at the same taxonomic levels were adjusted by FDR. Alpha diversity assessed the bacteria diversity within individuals was compared in different $H$. pylori infection status groups using Wilcoxon test. Beta diversity illustrating distance between individuals in terms of bacterial composition was demonstrated as principal coordinates analysis (PCoA) plot. Alpha and beta diversity analyses were conducted in R 3.6.2 with vegan ${ }^{71}$, phyloseq $^{72}$ and microbiome packages ${ }^{73}$. Additionally, we dichotomized the abundance data to represent whether a bacterium is present in an individual. Logistic regression adjusted for age and sex was then used to analyze the associations between the presence of bacteria and the status of $H$. pylori infection. FDR method was used for multiple testing adjustment. In pathway analysis, pathways present in over $10 \%$ of samples were selected for subsequent analyses.

Ethnics statement. The study protocol was reviewed and approved by the Institutional Review Board of BGI (No. BGI-IRB 16068). Review procedures in BGI-IRB meet GCP principles and relevant national regulations for this application. Written informed consent was obtained from all the study participants prior to their enrolment.

\section{Data availability}

The data supported the findings of this study has been deposited into CNGB Sequence Archive (CNGB) $)^{74}$ of China National GeneBank DataBase $(\mathrm{CNGBdb})^{75}$ with accession number CNP0001557.

Received: 26 May 2021; Accepted: 31 August 2021

Published online: 14 September 2021

\section{References}

1. Hooi, J. K. et al. Global prevalence of Helicobacter pylori infection: Systematic review and meta-analysis. Gastroenterology 153, 420-429 (2017).

2. Montecucco, C. \& Rappuoli, R. Living dangerously: How Helicobacter pylori survives in the human stomach. Nat. Rev. Mol. Cell Biol. 2, 457-466 (2001).

3. Kusters, J. G., Van Vliet, A. H. \& Kuipers, E. J. Pathogenesis of Helicobacter pylori infection. Clin. Microbiol. Rev. 19, 449-490 (2006).

4. Suerbaum, S. \& Josenhans, C. Helicobacter pylori evolution and phenotypic diversification in a changing host. Nat. Rev. Microbiol. 5, 441-452 (2007).

5. Berthenet, E. et al. A GWAS on Helicobacter pylori strains points to genetic variants associated with gastric cancer risk. BMC Biol. 16, 1-11 (2018).

6. Whitmire, J. M. \& Merrell, D. S. Helicobacter pylori genetic polymorphisms in gastric disease development. Adv. Exp. Med. Biol. 1149, 173-194 (2019).

7. Graham, D. Y. et al. Epidemiology of Helicobacter pylori in an asymptomatic population in the United States: Effect of age, race, and socioeconomic status. Gastroenterology 100, 1495-1501 (1991).

8. Malaty, H. M., Engstrand, L., Pedersen, N. L. \& Graham, D. Y. Helicobacter pylori infection: Genetic and environmental influences: a study of twins. Ann. Intern. Med. 120, 982-986 (1994).

9. Mayerle, J. et al. Identification of genetic loci associated with Helicobacter pylori serologic status. JAMA 309, 1912-1920 (2013).

10. Hansson, G. K. \& Edfeldt, K. Toll to be paid at the gateway to the vessel wall. Arterioscler. Thromb. Vasc. Biol. 25, 1085-1087 (2005).

11. Lagunes-Servin, H. et al. Toll-like receptors and cytokines are upregulated during Helicobacter pylori infection in Children. Helicobacter 18, 423-432 (2013). 
12. Simawaranon, T., Wattanawongdon, W. \& Tongtawee, T. Toll-like receptors are associated with Helicobacter pylori infection and gastric mucosa pathology. J. Clin. Microbiol. 10, e58351 (2017).

13. Falush, D. et al. Traces of human migrations in Helicobacter pylori populations. Science 299, 1582-1585 (2003).

14. Ma, J. et al. Associations between cytokine gene polymorphisms and susceptibility to Helicobacter pylori infection and Helicobacter pylori related gastric cancer, peptic ulcer disease: A meta-analysis. PLoS ONE 12, e0176463 (2017).

15. Negovan, A., Iancu, M., Fülöp, E. \& Bănescu, C. Helicobacter pylori and cytokine gene variants as predictors of premalignant gastric lesions. World J. Gastroenterol. 25, 4105 (2019).

16. Wang, D. et al. Alterations in the human gut microbiome associated with Helicobacter pylori infection. FEBS Open Bio 9, 1552-1560 (2019).

17. Gao, J.-J. et al. Association between gut microbiota and Helicobacter pylori-related gastric lesions in a high-risk population of gastric cancer. Front. Cell. Infect. Microbiol. 8, 202 (2018).

18. Marshall, C. R. et al. Structural variation of chromosomes in autism spectrum disorder. Am. J. Hum. Genet. 82, 477-488 (2008).

19. Kaminsky, E. B. et al. An evidence-based approach to establish the functional and clinical significance of copy number variants in intellectual and developmental disabilities. Genet. Med. 13, 777-784 (2011).

20. Gao, Y. et al. Long non-coding RNA FGD5-AS1 regulates cancer cell proliferation and chemoresistance in gastric cancer through miR-153-3p/CITED2 axis. Front. Genet. 11, 715 (2020).

21. Shimizu, T. et al. Accumulation of somatic mutations in TP53 in gastric epithelium with Helicobacter pylori infection. Gastroenterology 147, 407-417. e403 (2014).

22. Chang, Y.-C. et al. Common variation in the fat mass and obesity-associated (FTO) gene confers risk of obesity and modulates BMI in the Chinese population. Diabetes 57, 2245-2252 (2008).

23. Xu, X. et al. Relationship between Helicobacter pylori infection and obesity in Chinese adults: A systematic review with metaanalysis. PLoS ONE 14, e0221076 (2019).

24. Conteduca, V. et al. H. pylori infection and gastric cancer: State of the art. Int. J. Oncol. 42, 5-18 (2013).

25. Xu, D. et al. FTO expression is associated with the occurrence of gastric cancer and prognosis. Oncol. Rep. 38, 2285-2292 (2017).

26. Andersen, K. G. et al. Genome-wide scans provide evidence for positive selection of genes implicated in Lassa fever. Philos. Trans. R. Soc. Lond. B Biol. Sci. 367, 868-877 (2012).

27. Mootha, V. K. et al. PGC-1 $\alpha$-responsive genes involved in oxidative phosphorylation are coordinately downregulated in human diabetes. Nat. Genet. 34, 267-273 (2003).

28. Subramanian, A. et al. Gene set enrichment analysis: A knowledge-based approach for interpreting genome-wide expression profiles. Proc. Natl. Acad. Sci. U.S.A. 102, 15545-15550 (2005).

29. Guo, Y. et al. Effect of Helicobacter pylori on gastrointestinal microbiota: A population-based study in Linqu, a high-risk area of gastric cancer. Gut 69, 1598-1607 (2020).

30. Guzman-Murillo, M. A., Ruiz-Bustos, E., Ho, B. \& Ascencio, F. Involvement of the heparan sulphate-binding proteins of Helicobacter pylori in its adherence to HeLa S3 and Kato III cell lines. J. Med. Microbiol. 50, 320-329 (2001).

31. Epplein, M. et al. Helicobacter pylori prevalence and circulating micronutrient levels in a low-income United States population. Cancer Prev. Res. (Phila.) 4, 871-878 (2011).

32. Truong, D. T. et al. MetaPhlAn2 for enhanced metagenomic taxonomic profiling. Nat. Methods 12, 902-903 (2015).

33. Liu, S. et al. Altered gut microbiota and short chain fatty acids in Chinese children with autism spectrum disorder. Sci. Rep. 9, 1-9 (2019).

34. Zhai, S. et al. Dietary butyrate suppresses inflammation through modulating gut microbiota in high-fat diet-fed mice. FEMS Microbiol. Lett. 366, fnz153 (2019).

35. De Maesschalck, C. et al. Faecalicoccus acidiformans gen. nov., sp. nov., isolated from the chicken caecum, and reclassification of Streptococcus pleomorphus (Barnes et al. 1977), Eubacterium biforme (Eggerth 1935) and Eubacterium cylindroides (Cato et al. 1974) as Faecalicoccus pleomorphus comb. nov., Holdemanella biformis gen. nov., comb. nov. and Faecalitalea cylindroides gen. nov., comb. nov., respectively, within the family Erysipelotrichaceae. Int. J. Syst. Evol. Microbiol. 64, 3877-3884 (2014).

36. Kaakoush, N. O. Insights into the role of Erysipelotrichaceae in the human host. Front. Cell. Infect. Microbiol. 5, 84 (2015).

37. Brown, K., DeCoffe, D., Molcan, E. \& Gibson, D. L. Diet-induced dysbiosis of the intestinal microbiota and the effects on immunity and disease. Nutrients 4, 1095-1119 (2012).

38. Brahe, L. et al. Specific gut microbiota features and metabolic markers in postmenopausal women with obesity. Nutr. Diabetes $\mathbf{5}$, e159-e159 (2015).

39. Koh, A., De Vadder, F., Kovatcheva-Datchary, P. \& Bäckhed, F. From dietary fiber to host physiology: Short-chain fatty acids as key bacterial metabolites. Cell 165, 1332-1345 (2016).

40. Wang, L. et al. Acetic acid and butyric acid released in large intestine play different roles in the alleviation of constipation. J. Funct. Foods 69, 103953 (2020).

41. Corvaglia, L. et al. Accuracy of serology and 13C-urea breath test for detection of Helicobacter pylori in children. Pediatr. Infect. Dis. J. 18, 976-979 (1999).

42. Hess, M. E. \& Brüning, J. C. The fat mass and obesity-associated (FTO) gene: Obesity and beyond?. Biochim. Biophys. Acta Mol. Basis Dis. 1842, 2039-2047 (2014).

43. Loos, R. J. \& Yeo, G. S. The bigger picture of FTO-The first GWAS-identified obesity gene. Nat. Rev. Endocrinol. 10, 51-61 (2014).

44. Arslan, E., Atılgan, H. \& Yavaşoğlu, İ. The prevalence of Helicobacter pylori in obese subjects. Eur. J. Intern. Med. 20, 695-697 (2009).

45. Zhang, J. et al. Persistent infection of Helicobacter pylori affects weight loss in obese population compared with persistent negative: A case-control study based on healthy Chinese. Helicobacter 25, e12697 (2020).

46. Lin, B., Qing, X., Liao, J. \& Zhuo, K. Role of protein glycosylation in host-pathogen interaction. Cells 9, 1022 (2020).

47. Fransson, L. A. Structure and function of cell-associated proteoglycans. Trends Biochem. Sci. 12, 406-411 (1987).

48. Zhang, T., de Waard, A. A., Wuhrer, M. \& Spaapen, R. M. The role of glycosphingolipids in immune cell functions. Front. Immunol. $10,90(2019)$.

49. Mendz, G. L., Burns, B. P. \& Hazell, S. L. Characterisation of glucose transport in Helicobacter pylori. Biochim. Biophys. Acta Gen. Subj. 1244, 269-276 (1995).

50. Mendz, G. L., Hazell, S. L. \& Burns, B. P. The Entner-Doudoroff pathway in Helicobacter pylori. Arch. Biochem. Biophys. 312, 349-356 (1994).

51. Mendz, G. L., Hazell, S. L. \& Burns, B. P. Glucose utilization and lactate production by Helicobacter pylori. Microbiology 139, 3023-3028 (1993).

52. Marais, A., Mendz, G. L., Hazell, S. L. \& Mégraud, F. Metabolism and genetics of Helicobacter pylori: The genome era. Microbiol. Mol. Biol. Rev. 63, 642-674 (1999).

53. Som, S. et al. Mechanisms linking metabolism of Helicobacter pylori to ${ }^{18} \mathrm{O}$ and ${ }^{13} \mathrm{C}$-isotopes of human breath $\mathrm{CO}_{2}$. Sci. Rep. 5, 1-9 (2015).

54. Liu, Y. et al. Metabolic reprogramming results in abnormal glycolysis in gastric cancer: A review. Onco Targets Ther. 12, 1195 (2019).

55. Luo, B. et al. ATP-dependent Lon protease contributes to Helicobacter pylori-induced gastric carcinogenesis. Neoplasia 18, 242-252 (2016). 
56. Moyat, M. \& Velin, D. Immune responses to Helicobacter pylori infection. World J. Gastroenterol. 20, 5583 (2014).

57. Li, T. H. et al. Alterations in gastric microbiota after H. pylori eradication and in different histological stages of gastric carcinogenesis. Sci. Rep. 7, 1-8 (2017).

58. Durazzi, F. et al. Comparison between $16 \mathrm{~S}$ rRNA and shotgun sequencing data for the taxonomic characterization of the gut microbiota. Sci. Rep. 11, 1-10 (2021).

59. Evrengul, H. et al. Elevated homocysteine levels in patients with slow coronary flow: Relationship with Helicobacter pylori infection. Helicobacter 12, 298-305 (2007).

60. Rossi, M., Amaretti, A. \& Raimondi, S. Folate production by probiotic bacteria. Nutrients 3, 118-134 (2011).

61. Li, H. \& Durbin, R. Fast and accurate short read alignment with Burrows-Wheeler transform. Bioinformatics 25, 1754-1760 (2009).

62. DePristo, M. A. et al. A framework for variation discovery and genotyping using next-generation DNA sequencing data. Nat. Genet. 43, 491-498 (2011).

63. Purcell, S. et al. PLINK: A tool set for whole-genome association and population-based linkage analyses. Am. J. Hum. Genet. 81, 559-575 (2007)

64. Balding, D. J. A tutorial on statistical methods for population association studies. Nat. Rev. Genet. 7, 781-791 (2006).

65. Pruim, R. J. et al. LocusZoom: Regional visualization of genome-wide association scan results. Bioinformatics 26, 2336-2337 (2010).

66. Neale, B. M. \& Sham, P. C. The future of association studies: Gene-based analysis and replication. Am. J. Hum. Genet. 75, 353-362 (2004).

67. Yang, J., Lee, S. H., Goddard, M. E. \& Visscher, P. M. GCTA: A tool for genome-wide complex trait analysis. Am. J. Hum. Genet. 88, 76-82 (2011).

68. Bakshi, A. et al. Fast set-based association analysis using summary data from GWAS identifies novel gene loci for human complex traits. Sci. Rep. 6, 1-9 (2016).

69. Li, R. et al. SOAP2: An improved ultrafast tool for short read alignment. Bioinformatics 25, 1966-1967 (2009).

70. Franzosa, E. A. et al. Species-level functional profiling of metagenomes and metatranscriptomes. Nat. Methods 15, 962-968 (2018).

71. Oksanen, J. et al. vegan: Community Ecology Package. R package version 2.5-7, https://CRAN.R-project.org/package=vegan (2020).

72. McMurdie, P. J. \& Holmes, S. phyloseq: An R package for reproducible interactive analysis and graphics of microbiome census data. PLoS ONE 8, e61217 (2013).

73. Lahti, L. \& Shetty, S. Tools for microbiome analysis in R. Version, http://microbiome.github.com/microbiome (2017).

74. Guo, X. et al. CNSA: A data repository for archiving omics data. Database (Oxford) https://doi.org/10.1093/database/baaa055 (2020).

75. Chen, F. Z. et al. CNGBdb: China National GeneBank DataBase. Yi Chuan 42, 799-809 (2020).

\section{Acknowledgements}

We thank for the funding support from Shenzhen Municipal Government of China (No. JCYJ20170412153155228).

\section{Author contributions}

Y.L. and C.N. designed the study, D.X.H. and Y.Q.L. analysed the data. D.X.H., Y.Q.L., D.M.W. and Y.L. interpreted the data and wrote the manuscript. All authors read and approved the final version of the manuscript.

\section{Competing interests}

The authors declare no competing interests.

\section{Additional information}

Supplementary Information The online version contains supplementary material available at https://doi.org/ 10.1038/s41598-021-97790-7.

Correspondence and requests for materials should be addressed to C.N. or Y.L.

Reprints and permissions information is available at www.nature.com/reprints.

Publisher's note Springer Nature remains neutral with regard to jurisdictional claims in published maps and institutional affiliations.

(c) (i) Open Access This article is licensed under a Creative Commons Attribution 4.0 International License, which permits use, sharing, adaptation, distribution and reproduction in any medium or format, as long as you give appropriate credit to the original author(s) and the source, provide a link to the Creative Commons licence, and indicate if changes were made. The images or other third party material in this article are included in the article's Creative Commons licence, unless indicated otherwise in a credit line to the material. If material is not included in the article's Creative Commons licence and your intended use is not permitted by statutory regulation or exceeds the permitted use, you will need to obtain permission directly from the copyright holder. To view a copy of this licence, visit http://creativecommons.org/licenses/by/4.0/.

(C) The Author(s) 2021 